\title{
Carbohydrates intake for a healthy diet
}

\begin{abstract}
The science of nutrition is continuously evolving. For many decades the attention in releasing dietary guidelines was focused mainly on calories and their distribution among the three macronutrients: protein, carbohydrates and fats. Currently nutritional sciences rely on a greater amount of findings and information, including those about the effects of nutrients on genes' expression and hormones, and the increased importance of the wide variety of micronutrients contained in fresh, whole, and natural foods, such as fruits, vegetables, and spices. For example, Chowanadisai et al.1 showed the remarkable ability of pyrroloquinoline quinone to stimulate the mitochondrial biogenesis, and to positively modulate gene expression. Foods and nutrients affect body composition and health not only by supplying calories, but in many complex ways, including changes of several hormones' levels. For example, although sucrose does not contain any fat or cholesterol, it is considered to be an unhealthy food, to be consumed in limited amounts, because of its effects on insulin levels. Insulin spikes can impair health and promote cardiovascular diseases by stimulating lipogenesis, increasing body fat, triglycerides, and pro-inflammatory molecules. Starch sources, such as bread, rice, and pasta, can increase blood glucose levels more than sucrose. Using starch as the main dietary source of energy might represent a relevant pejorative factor for human health and longevity, which could contribute to the development of some of the most common chronic diseases. Healthy fats, on the contrary, might be a better choice as main source of energy, provided carbohydrates and insulin levels are kept under strict control.
\end{abstract}

Keywords: carbohydrates, fats, glycemic index, calories, hormones, inflammation, heart diseases, cholesterol, nutrition
Volume 3 Issue 3 - 2016

\section{Nicola Di Pietro}

Università Cattolica del Sacro Cuore, Canada

Correspondence: Nicola Di Pietro, Università Cattolica del Sacro Cuore, Canada, Email nikodipietro@gmail.com

Received: December 30, 2015 | Published: December 30, 2016

\section{Abbreviations: GI, glycemic index; g, grams; EPA, eicosapentaenoic acid}

\section{Introduction}

Nutritional sciences are dramatically evolving. The effects of foods and nutrients on hormones have a great importance and relevance, and have to be considered while designing dietary guidelines for healthy eating. Hormones are able to determine the destiny of ingested calories and nutrients, including whether they have to be oxidized as energy or stored as deposit. Insulin is one of the most relevant hormones responding to ingested food, especially in regard to fat metabolism. It has a powerful lipogenic and antilipolytic effect in human adipose tissue. High insulin level might also be detrimental on health, especially under a cardiovascular point of view. Indeed, insulin spikes can increase the levels of endogenous cholesterol, and of cellular mediators causing inflammation, blood coagulation, vasoconstriction, autoimmune reactions, and asthma. High insulin spikes are caused by dietary sugars. Furthermore, sugars also damage the vascular endothelium and deplete its nitric oxide levels. Complex carbohydrates, mainly starches from grains, have always been recommended as main energy source of the diet, in amounts 5 to 30 times higher than sucrose, even though they are nutrients of the same type, which is pure carbohydrates containing neither fats nor cholesterol. This occurred because of a presumed slower absorption speed of starch. However, the assessment of the glycemic index of foods showed that they exert at least the same impact on blood glucose as sucrose does, even higher in many cases. The usually recommended daily intake of complex carbohydrates might have similar effects on the body as those associated with the ingestion of $200 \mathrm{~g}$ to $400 \mathrm{~g}$ of sucrose every day. Healthy fatty acids might be the best energy source for the body, provided a strict control on carbohydrates ingestion and blood insulin levels.

\section{Hormones}

Nutrients can affect the levels of important hormones, such as glucagon, insulin, cortisol, etc. Hormones can determine the destiny of nutrients and calories ingested with food, affecting their oxidation into energy or accumulation. Furthermore, a single hormone, such as insulin, can affect the levels of several other hormones and cellular mediators, causing a cascade of effects in the body. It is important to consider these effects in order to determine their recommended daily intakes.

\section{Calcium and bones}

Calcium is a clear example of nutrient whose storage in the bones is determined by hormones. Calcitonin, parathormone, and vitamin $\mathrm{D}$ are hormones that regulate blood levels, bone release and storage of calcium. A severe lack of vitamin D could decrease bone density independently of the dietary calcium intake. It causes osteomalacia in adults and rickets in infants. ${ }^{2}$

\section{Proteins and muscle mass}

Muscle tissue is made of protein. The increased hypertrophy and growth caused by the administration of anabolic steroids ${ }^{3}$ is another example of increased storage of nutrients caused by hormones (amino acids in form of proteins in this case). 


\section{Adipose tissue}

Like muscle mass, adipose tissue is also influenced by the action of hormones. Thyroid hormones, for example, stimulate fat oxidation, ${ }^{4}$ while insulin increases fat deposit. Similarly to what happens to muscle mass with an increase of anabolic hormones, an increase of insulin can lead to increased body fat. This is very important, especially in regard to carbohydrate ingestion.

\section{Effects of Insulin}

Several are the effects of insulin on body fat and functioning. On adipose tissue it exerts a "powerful" antilipolytic and lipogenic action; ${ }^{5-7}$ it is able to increase by $2000-3000 \%$ the transportation of glucose in the adipocites stimulating the GLUT-4 enzyme, which is involved in fat uptake. ${ }^{5,6}$ At this regard, Korean researchers ${ }^{8}$ compared the effects of three different types of meals on the ability of the body to oxidize fats during physical exercise. The meal of one group consisted in water. The other two groups received two different isocaloric meals, with the same amount of carbohydrates, but different glycemic index: a high and a low glycemic index type of carbohydrate respectively. The group ingesting the low glycemic index carbohydrates showed a similar fat oxidation as the water only group, while the high glycemic index carbohydrates inhibited the fat oxidation of the respective group. Since calories were the same, and the low glycemic index meal showed a similar response to water, the fat-burning impairment of the third group was most likely due to the insulin spike induced by the post-prandial glucose spike of the high glycemic index carbohydrates.

Insulin can also increase the synthesis of cholesterol, ${ }^{9-11}$ which has a particular relevance, since the endogenous cholesterol represents the main quota in the blood, up to $80 \% .^{11,12}$ Insulin can widely affect the biochemistry of the body. It is able to activate the Delta5 -Desaturase enzyme, ${ }^{13,14}$ which increases the levels of Arachidonic Acid. Arachidonic acid is the precursor of several pro-inflammatory prostaglandins, pro-coagulant thromboxanes, and leukotrienes which can be involved in cardiovascular diseases, autoimmune reactions and asthma. ${ }^{11,15}$ Preventing inflammation is of paramount importance for chronic diseases, such as cardiovascular and neurological diseases; assuring a proper vasodilation and blood fluidity is also very important for preventing or managing the mentioned disorders. For all the reasons mentioned above it is clear that preventing sharp blood glucose spikes should be considered as one of the most important strategies to consider when implementing dietary recommendations.

\section{Recommended carbohydrates intake}

In past one of the main factors that was considered in order to establish the recommended carbohydrate amounts for a healthy diet was their chemical structure. Since the intestine can only absorb monosaccharides, it was supposed that the more the structure of a carbohydrate was "complex", the slower its absorption speed would have been. Thus, polysaccharides have been defined "complex carbohydrates", whereas mono- and di-saccharides have been defined simple sugars. This concept leads to specific and substantial characteristics of the so-called food pyramid.

\section{The food pyramid}

This visual tool shows on top, the apex, foods that are more likely to be unhealthy, and that have to be consumed in very moderate amounts. Simple sugars are included among them. The more the pyramid structure enlarges towards its base, the healthier and safer are considered the foods that have been assigned to any determined level. These foods have to be ingested in larger amounts: the larger is the level of the food pyramid to which foods are assigned, the larger are their recommended dietary amounts. Complex carbohydrates are considered the main macronutrient of a healthy diet. For this reason, grain-derived foods, rich on starch, a complex carbohydrate (such as pasta, rice, bread, and cornflakes) have been placed at the base of the food pyramid, the larger level. ${ }^{16}$ In some cases they have been replaced with vegetables and moved up to the second level, ${ }^{17}$ which still represents foods considered healthy and safe if consumed in relatively large amounts.

\section{Complex carbohydrates}

In the Food Pyramid published by the U.S. Office of Disease Prevention and Health Promotion, ${ }^{16}$ for example, the recommended daily servings of the group including bread, pasta, rice, and cereals are between 6 and 11. In terms of grams of carbohydrates, they range between approximately $215 \mathrm{~g}$ and $400 \mathrm{~g}$ of complex carbohydrates per day.

The Italian Istituto di Scienza dell'Alimentazione placed bread, pasta, rice, cookies, and potatoes on the second level of the food pyramid. ${ }^{17}$ The amount of net complex carbohydrates contained in the institution's recommended weekly amounts of bread, pasta, rice, baked products, and potatoes for an average healthy diet ${ }^{18}$ can be calculated as approximately $1230 \mathrm{~g}$. It corresponds to a daily amount of 175g. Cappelli et al., ${ }^{19}$ for an average well-balanced diet, recommend to ingest between $400 \mathrm{~g}$ and $550 \mathrm{~g}$ of carbohydrates per day, of which no more than $60-70 \mathrm{~g}$ from all the sources of simple sugars (including sucrose, honey, fruit, etc.), which means a net amount of complex carbohydrates included between 330 and $490 \mathrm{~g}$.

\section{Detrimental effects of simple sugars}

In the above mentioned American food pyramid, ${ }^{16}$ the simple sugars are placed on top, represented as added sugars, in the category named "use sparingly". The Italian food pyramid ${ }^{17}$ places sucrose and honey on top of the pyramid. The recommended daily amount of sucrose consists in $15 \mathrm{~g}$ per day ( 3 portions of $5 \mathrm{~g}$ each). ${ }^{20}$

Simple sugars and starches are the same type of nutrient: carbohydrates. Their calorie intake is equal, but it is unthinkable to switch their intake, in a way to ingest approximately 15 to $30 \mathrm{~g}$ /day of starches and 200 to $400 \mathrm{~g}$ /day of sucrose, even combining fats, fibres and other foods in order to slow down its absorption. And yet sucrose, as well as starch, doesn't contain fats of any kind, neither cholesterol. It is a pure carbohydrate source.

The reason why it is recommended to dramatically limit the amounts of sucrose lies in its effects in the body, rather than in a high content of cholesterol or other detrimental molecules. By being a simple sugar, its absorption speed is very high. Once eaten, it causes very sharp blood glucose spikes and, consequently, insulin spikes. A chronic daily intake of sucrose above the recommended limits, would be likely to cause the harmful effects mentioned above, in the paragraph dedicated to the effects of insulin in the body: lipogenic and antilipolytic effect, weight gain, increased inflammation, blood coagulation, vasoconstriction, risk of cardiovascular diseases, autoimmune reactions, asthma, etc. Talking about a well-balanced diet, Cappelli et al., ${ }^{19}$ for example, suggest to focus on cornflakes or crisp bread for breakfast rather than on jam and sugar, because the carbohydrates of the firsts are absorbed slowly and increase the blood 
glucose moderately, without causing deleterious sharp blood insulin spikes. Again, the authors state that the complex carbohydrates have to be "undoubtedly" preferred to simple sugars, using a 5:1 ratio.

Furthermore, blood glucose spikes can also affect the vascular endothelial function, oxidize the vascular endothelium, increase the inflammatory reactions and the likelihood of the occurrence of cardiovascular diseases, ${ }^{21,22}$ prevent the arterial dilation, ${ }^{23}$ and inactivate nitric oxide. ${ }^{22,24}$ Among all these effects, the nitric oxide depletion alone would already be a relevant reason of concern in case of consumption of amounts of sucrose higher than those recommended. It indeed is required to regulate the elasticity of vessels, the inflammatory processes, ${ }^{25}$ to protect against atherosclerosis and cardiovascular diseases,,$^{25,26}$ and for many other processes.

\section{Glycemic index}

The assumption that complex carbohydrates are absorbed slowly because the intestine can only absorb monosaccharides has been tested since many decades. It has been shown that the behaviour of most starch sources doesn't match the expectations. Cappelli et al. ${ }^{19}$ report that the glycemic index (GI) of sucrose is 59, whereas that of white bread is 69 , and the GI of cornflakes is 80 . The University of Sydney created a website ${ }^{27}$ containing an extensive database of the GI of many different food products and varieties. The Table 1 shows the GI of some of the most common foods containing complex carbohydrates.

Among the main dietary starch sources, the products with the less high GI are certain varieties of pasta, especially spaghetti. Other varieties have the same impact of sucrose on blood glucose. On average the effect of pasta is slightly lower but similar to that of sucrose. All the other food products of the mentioned category have a higher IG.

Table I Glycemic index of common food sources of starch. ${ }^{27}$

\begin{tabular}{ll}
\hline Food products & Glycemic index \\
\hline Sucrose (25g) & 60 \\
Pasta (Spaghetti, white, boiled) & 41 \\
Pasta (Fusilli, boiled in salted water) & 61 \\
Corn flakes ${ }^{\mathrm{TM}}$ (average) & 78 \\
$\begin{array}{l}\text { Rice (Medium Grain rice, white, Double Ram } \\
\text { brand - average) }\end{array}$ & 82 \\
Bread (Whole wheat, French) & 85 \\
Bread (Italian, white) & 89 \\
\hline
\end{tabular}

\section{Same as sugar}

The meaning of these results is very important. They show that a given amount of complex carbohydrates from starch has at least the same effect on blood glucose in the human body as the same amount of sucrose, and most of the times an even higher impact. Eating $215 \mathrm{~g}$ or $400 \mathrm{~g}$ of starch every day, as recommended by the American food pyramid, ${ }^{16}$ might produce the same effects in the body as eating at least 215 or $400 \mathrm{~g}$ of sucrose respectively, which means approximately 40 or 80 teaspoons, every day. A similar comparison applies to the Italian food pyramid and dietary guidelines shown above. The irrelevant difference between the GI of white and whole bread shown in Table
1 also suggests that a few grams of fibers that can be added to a meal by choosing whole grain products, or adding some vegetables, are not enough to make hundreds of grams of sucrose-like carbohydrates (starches) better than the recommended $15 \mathrm{~g}$ limit established for sucrose. Neither it is advisable to eat 100 or $150 \mathrm{~g}$ of sucrose in a single meal, just because of the GI decrease caused by the combination of fats and other foods together. If this type of nutrition can have effects similar to that of the chronic daily ingestion of $40 \mathrm{~g}$ to 80 teaspoons of sucrose, it is not a coincidence that diabetes, cardiovascular, and brain diseases present a worrisome diffusion.

\section{Conclusion}

At the question "What if I want to lose or gain weight?" the United States Department of Agriculture replies that "the best and simplest way to lose weight" is to increase the physical activity "and reduce the fat and sugars" in the diet. ${ }^{28}$ If the impact of starch is similar to that of sucrose, complex carbohydrates should most probably be reduced accordingly.

The lipogenic and antilipolytic effect of insulin impairs a proper utilization of fats. If starches from grains would be considered similar to sucrose, and dramatically decreased to similar amounts, the human body might probably be able to utilize much better the dietary fats as energy source. Fats could be the actual best source of energy for a good health and efficiency, providing a very strict control on carbohydrates intake and blood insulin levels.

It would be very interesting to further investigate the metabolic and cardiovascular behavior of the body undergoing a regular, stable, and well-balanced mid- to long-term lower-carbohydrate and higherfat type of diet. The daily intake of carbohydrates could be established as just enough to prevent ketosis, making sure to choose low-glycemic index foods as sources and to divide it throughout all the meals and snacks, in order to never ingest more than 20 to $30 \mathrm{~g}$ of carbohydrates per time. Furthermore, the body can produce glucose from fats, and the brain doesn't need insulin in order to uptake from the blood the glucose it needs.

The fat sources should also be chosen properly, since an excess of Omega-6 fatty acids might interfere with the assessment of possible benefits of the proposed regimen. The dietary fats should be sourced in a way to simulate a natural wild environment, primarily from grass-fed meat, fish, olives, almonds, and nuts. The goal should be to provide mainly monounsaturated fatty acids as energy source, and to restore as more as possible the primordial Omega-6: Omega-3 fatty acids ratio. The Omega-3 fatty acid EPA (Eicosapentaenoic Acid) is able to counteract the effects of glucose and insulin on the increase of inflammatory molecules (Sears, 2003).

An optimized utilization of resources and spaces, an intelligent distribution, diffusion, and integration of pastures and grass, and a stronger adoption of special techniques, such as vertical farming and hydroponics, should be included in a really effective strategy to heal the world.

Grains are basically a source of sugar in form of starch; they don't even contain relevant amounts of high quality proteins and micronutrients, such as vitamins, minerals, polyphenols, and other phytonutrients, like fish, grass-fed meat, fresh vegetables and fruits do. Grains might not be the right answer to world hanger. They might mean feeding the world on sugar. 


\section{Acknowledgements}

None.

\section{Conflict of interest}

The author declares no conflict of interest

\section{References}

1. Chowanadisai W, Bauerly KA, Tchaparian E, et al. Pyrroloquinoline quinone stimulates mitochondrial biogenesis through cAMP response element-binding protein phosphorylation and increased PGC-1alpha expression. J Biol Chem. 2010;285(1):142-152.

2. NIH. Vitamin D-Fact Sheet for Health Professionals. USA: National Institute of Health; 2014.

3. Hartgens F, Kuipers H. Effects of androgenic-anabolic steroids in athletes [Abstract]. Sports Medicine. 2004;34(8):513-554.

4. Moreno M, de Lange P, Lombardi A, et al. Metabolic effects of thyroid hormone derivatives. Thyroid. 2008;18(2):239-253.

5. Pankov YA. Adipogenic function and other biologic effects of insulin. Bio meditsinskaia khimiia. 2016;62(1):5-13.

6. Arienti G. Le basi molecolari della nutrizione. Padova, Italy: Piccin Nuova Libraria; 2000.

7. Siliprandi N, Tettamanti G. Biochimica medica. $2^{a}$ edizione interamente rielaborata. Padova, Italy: Piccin Nuova Libraria; 1990.

8. Brainum J. Applied metabolics. Rendiamo utile la scienza. Vol 1, Figline Valdarno, Italy: Sandro Ciccarelli Editore; 1999.

9. Osborne AR, Pollock VV, Lagor WR, et al. Identification of insulinresponsive regions in the HMG-CoA reductase promoter. Biochemical and Biophysical Research Commununications. 2004;318(4):814-818.

10. Stefani M. Biochimica. Con elementi di biologia molecolare e scienze dell'alimentazione. Bologna, Italy: Zanichelli; 1997.

11. Sears B, Ottoz E. Come raggiungere la zona. Milano, Italy: Spearling \& Kupfer Editori; 1997.

12. Perlmutter D, Loberg K. Grain Brain. NY. Little, New York, USA: Brown and Company; 2013.

13. Sears B, Ricordi C. Anti-inflammatory nutrition as a pharmacological approach to treat obesity. Journal of Obesity. 2011:431985.
14. el Boustani S, Causse JE, Descomps B, et al. Direct in vivo characterization of delta 5 desaturase activity in humans by deuterium labeling: effect of insulin. Metabolism: Clinical and Experimental.1989;38(4):315-321.

15. Sears B. La zona omega 3 RX. Milano, Italy: Spearling \& Kupfer Editori; 2003.

16. Dietary Guidelines-Build a Healthy Base. U.S. Office of disease prevention and health promotion, USA; 2000.

17. Piramide Giornaliera. Italy: Istituto di scienza dell'alimentazioneUniversità La sapienza di roma; 2005,

18. Piramide alimentare. Italy: Istituto di scienza dell'alimentazioneUniversità La sapienza di roma; 2005.

19. Cappelli P, Vannucchi V. Complementi di scienza dell'alimentazione. Zanichelli, Bologna, Italy; 2000.

20. Dolci zucchero e miele. Italy: Retrieved from Istituto di scienza dell'alimentazione-Università La sapienza di roma; 2005.

21. Mah E, Noh SK, Ballard KD, et al. Supplementation of a gammatocopherol-rich mixture of tocopherols in healthy men protects against vascular endothelial dysfunction induced by postprandial hyperglycemia. J Nutr Biochem. 2012;24(1):196-203.

22. Ceriello A, Taboga C, Tonutti L, et al. Evidence for an independent and cumulative effect of postprandial hypertriglyceridemia and hyperglycemia on endothelial dysfunction and oxidative stress generation: effects of shortand long-term simvastatin treatment. Circulation. 2002;106(10):12111218 .

23. Kawano H, Motoyama T, Hirashima O, et al. Hyperglycemia rapidly suppresses flow-mediated endothelium-dependent vasodilation of brachial artery. J Am Coll Cardiol. 1999;34(1):146-154.

24. Ogita H, Liao J. Endothelial function and oxidative stress. Endothelium. 2004;11(2):123-132.

25. Shaw CA, Taylor EL, Megson IL, et al. Nitric oxide and the resolution of inflammation: implications for atherosclerosis. Memorias dó Instituto Oswaldo Cruz. 2005;100(1).

26. Shaul PW. Endothelial nitric oxide synthase, caveolae and the development of atherosclerosis. J Physiol. 2003;547(Pt 1):21-33.

27. Glycemic Index. (n.d.). Australia: University of Sydney; 2017.

28. The Food Guide Pyramid. USA: United States Department of Agriculture; 1996. 